\title{
CARDIFRC $^{\circledR}-$ Development and mechanical properties. Part II: Fibre distribution
}

\author{
S. D. P. Benson* $†$ D. Nicolaides* and B. L. Karihaloo*
}

Cardiff University

Part II of this three-part paper deals with the assessment of the distribution of fibres in CARDIFRC ${ }^{\circledR}$, a class of high-performance short steel fibre-reinforced cementitious composites. The effect of the mixing procedure and of the shape of the object cast from CARDIFRC $C^{\mathbb{B}}$ on the distribution of the fibres is assessed using both a nondestructive and a destructive technique. The non-destructive technique is based on computerised tomography imaging and the destructive technique on image analysis.

\section{Introduction}

The mechanical performance of any highperformance, fibre-reinforced cementitious composite (HPFRCC), of which CARDIFRC ${ }^{\circledR}$ is a patented example, depends to a large extent on the even distribution of fibres in the bulk of the material. Any regions with a low density of fibres or with no fibres are potential sites of weakness. The distribution of fibres in the mix depends not only on how the fibres were introduced into the mix but also on the shape of the object cast from CARDIFRC ${ }^{\circledR}$.

In order to ensure that the mixing and compaction procedures used to make the matrix do indeed result in an even and random distribution of fibres in the hardened material both non-destructive [computerised tomography (CT) imaging $^{1}$ ] and destructive (image analysis) techniques were employed. Various specimens cast from the matrix (tensile and bend specimens, cylinders and thin strips) were examined using both techniques to reveal the effect of the shape of the specimen on the fibre distribution. The aim of trying two techniques was to check if their results are in some way correlated, because that would open a quick and efficient route to testing the quality of industrially

\footnotetext{
* School of Engineering, Queen's Buildings, Newport Road, PO Box 925, Cardiff, CF24 0YF, UK.

$\uparrow$ Present address: Parsons Brinckerhoff, 29 Cathedral Road, Cardiff CF11 9HA, UK.
}

(MCR 41271) Paper received 22 April 2004; last revised 14 October 2004; accepted 1 February 2005 produced CARDIFRC ${ }^{\circledR}$ products using only the CT imaging technique.

\section{Test specimens}

Mixes of Types I and II were prepared according to the patented procedure ${ }^{2}$ (Part I) and were cast in moulds to form beams $(100 \mathrm{~mm} \times 100 \mathrm{~mm}$ $\times 500 \mathrm{~mm})$, cylinders $(100 \mathrm{~mm} \times 200 \mathrm{~mm})$ and thin strips $(250 \mathrm{~mm} \times 100 \mathrm{~mm} \times 16$ or $20 \mathrm{~mm})$. The moulds were filled in two or three layers depending upon specimen thickness and vibrated on a non-magnetic table at $50 \mathrm{~Hz}$. Prior to adding each additional layer the mix was fully vibrated to expel trapped air bubbles and lightly tamped to aid workability. When fully vibrated the surface was smoothed over with a float. For the specimens cast, standard 28-day curing at $20^{\circ} \mathrm{C}$ and accelerated hot curing ${ }^{3,4}$ have been tried and found to lead to almost identical mechanical properties. In the accelerated hot-curing process the specimens are left for $24 \mathrm{~h}$ before demoulding. They are then transferred to a water-curing tank at $20^{\circ} \mathrm{C}$. Over a $24 \mathrm{~h}$ period the temperature is gradually increased to $90^{\circ} \mathrm{C}$. During this temperature rise phase the material is subjected to varied expansions due to different coefficients of expansion of the various mix constituents. Note that it is important that with the increase in temperature the stress gradients are kept to a minimum as the constituents closer to the heat source will be heated more quickly than the core of the specimen and therefore stress gradients will arise, which will result in thermal shock cracking. The maximum temperature of $90^{\circ} \mathrm{C}$ is maintained for 7 days before cooling the specimens. 
The cooling phase, like the temperature rise phase, should be gradual. Although the concrete has gained in strength and is therefore better able to take up the stresses, the rate of cooling should be over a $24 \mathrm{~h}$ period to avoid cracking. Cracks are likely to occur when the difference between the temperatures of the core and the surface is greater than $15^{\circ} \mathrm{C} .{ }^{3}$ Hot curing thus decreases the curing time from 28 days to 9 days which maybe an advantage in the industrial production of CARDIFRC ${ }^{\circledR}$ products.

\section{Non-destructive CT imaging}

For non-destructive evaluation of the fibre distribution, computerised tomography (CT) imaging technique was employed using the latest General Electric CTi Helical Scanner at Velindre Hospital, Cardiff. ${ }^{5}$ CT imaging is a technique whereby cross-sectional images are generated by computer software from multiple X-ray readings. In the latest generation of helical scanners, the X-ray tube and detector are continuously rotated in a stator around the object being scanned, and the object is moved forward continuously through the centre of the stator. The amount of the X-ray beam transmitted through the object is measured, in all directions around the $360^{\circ}$ stator, and the X-ray attenuation, measured in Hounsfield units, calculated. The images are compiled from thousands of readings taken in the different directions using a computer program based on an image reconstruction algorithm. The idea of using CT imaging was first introduced for plain concrete by Karihaloo and Jefferson ${ }^{1}$ and has been extended to study the influence of mixing and compaction procedures on the distribution of fibres in various specimens cast from CARDIFRC $^{\circledR}$.

The CT images are produced by mapping the X-ray absorption density in Hounsfield units on to a grey scale, such that air appears black $(-1000$ Hounsfield units) and the densest particles in the specimen appear white (3017 Hounsfield units) with water being calibrated at 0 Hounsfield units. The resolution of detail in a medical CT scanner depends upon the selected field of view and image matrix size shown, as well as how finely the views are sampled. In the present case, a $512 \times 512$ matrix was used for a $150 \mathrm{~mm}$ wide field of view, giving an in-plane resolution of $0.29 \mathrm{~mm}$. Thus as even the latest CT helical scanners are unable to resolve individual steel fibres because of their small diameter $(0.16 \mathrm{~mm})$, it was decided to produce contour plots of the X-ray absorption density at two or three sections along the length of each specimen. The specimen could subsequently be cut along these sections and the fibre distribution analysed by image analysis. In this way, the X-ray absorption density contours can be correlated to the actual fibre distribution. ${ }^{5}$

In order to produce the contour plots, a $10 \mathrm{~mm} \times 10 \mathrm{~mm}$ grid was overlaid on the selected scanned sections on the computer screen and a probe was used to measure the X-ray absorption density at each grid intersection point, whose exact co-ordinates along the cross-section were also recorded. From these measurements contour plots of X-ray absorption density along the selected cross-sections were constructed using the software SURFER 8. Figs 1-4 show the locations of the sections at which the specimens were scanned, together with the X-ray absorption density grey scale images and the contour plots along two selected cross-sections of each specimen. It will be noticed that dark spots in the grey scale images correspond to a lower X-ray absorption density on the contour plots; that is, to a lower fibre concentration. These figures demonstrate that the fibre distribution is generally uniform, irrespective of the shape of the specimen made from CARDIFRC ${ }^{\circledR}$.

\section{Destructive image analysis}

The locations of the sections scanned by the CT scanner were carefully marked on the specimens. The specimens were cut along these marked locations (Fig. 5) using a wet-cut diamond blade circular saw (3 mm thick blade). The section was placed on the photographic light table and a $10 \mathrm{~mm} \times 10 \mathrm{~mm}$ grid (Fig. 6) was placed on top to accurately position the section under the camera. Each square of the grid was numbered and examined thoroughly under the microscope. After positioning the section to capture the selected square for fibre counting, the grid was removed. The light intensity and the position of the microscope lens were adjusted to achieve the most vivid image of the examined square of the grid on the monitor, and the points where fibres appeared in the square were marked by white spots. The rest of the examined grid square was painted black on the image. A charge-coupled device (CCD) camera coupled with a $80 \mathrm{~mm}$ optical lens was used to acquire the image (Fig. 7). This image was processed by appropriate image analysis software (Leica Quantimet 500), which categorised the fibres by taking an average of four measurements to determine their equivalent diameter and placing this value in the appropriate range. The ranges selected were based on the fibre diameter $(0 \cdot 16 \mathrm{~mm})$ and were set at $0 \cdot 16-$ $0.32 \mathrm{~mm}, 0.32-0.48 \mathrm{~mm}$, etc. The range $0-0.16 \mathrm{~mm}$ was omitted. The smallest possible fibre image that can be seen after cutting (which corresponds to a fibre that is perpendicular to the cut section) is $0.16 \mathrm{~mm}$, irrespective of the angle at which the fibre is embedded in the matrix. Therefore, if a feature with an equivalent diameter smaller than $0.16 \mathrm{~mm}$ is captured it cannot be a fibre; the inclusion of the range $0-0 \cdot 16 \mathrm{~mm}$ would lead therefore to an erroneous fibre count. For this reason, there is no point in increasing the resolution of the image. This procedure was repeated for the whole grid overlaid on each selected section, and the number 


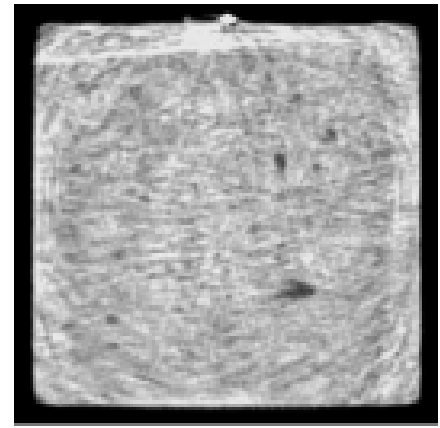

(a)

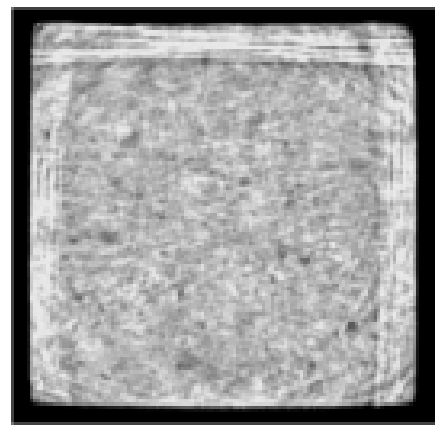

(c)

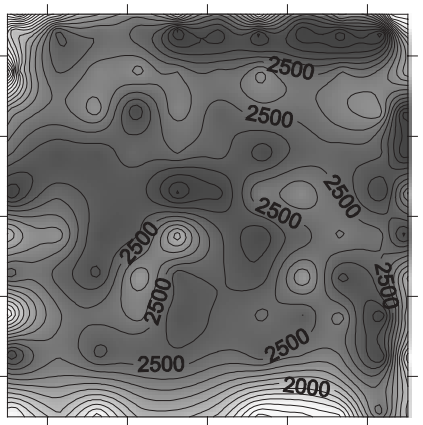

(b)

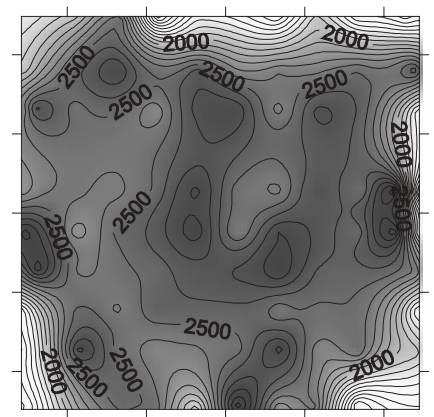

(d)
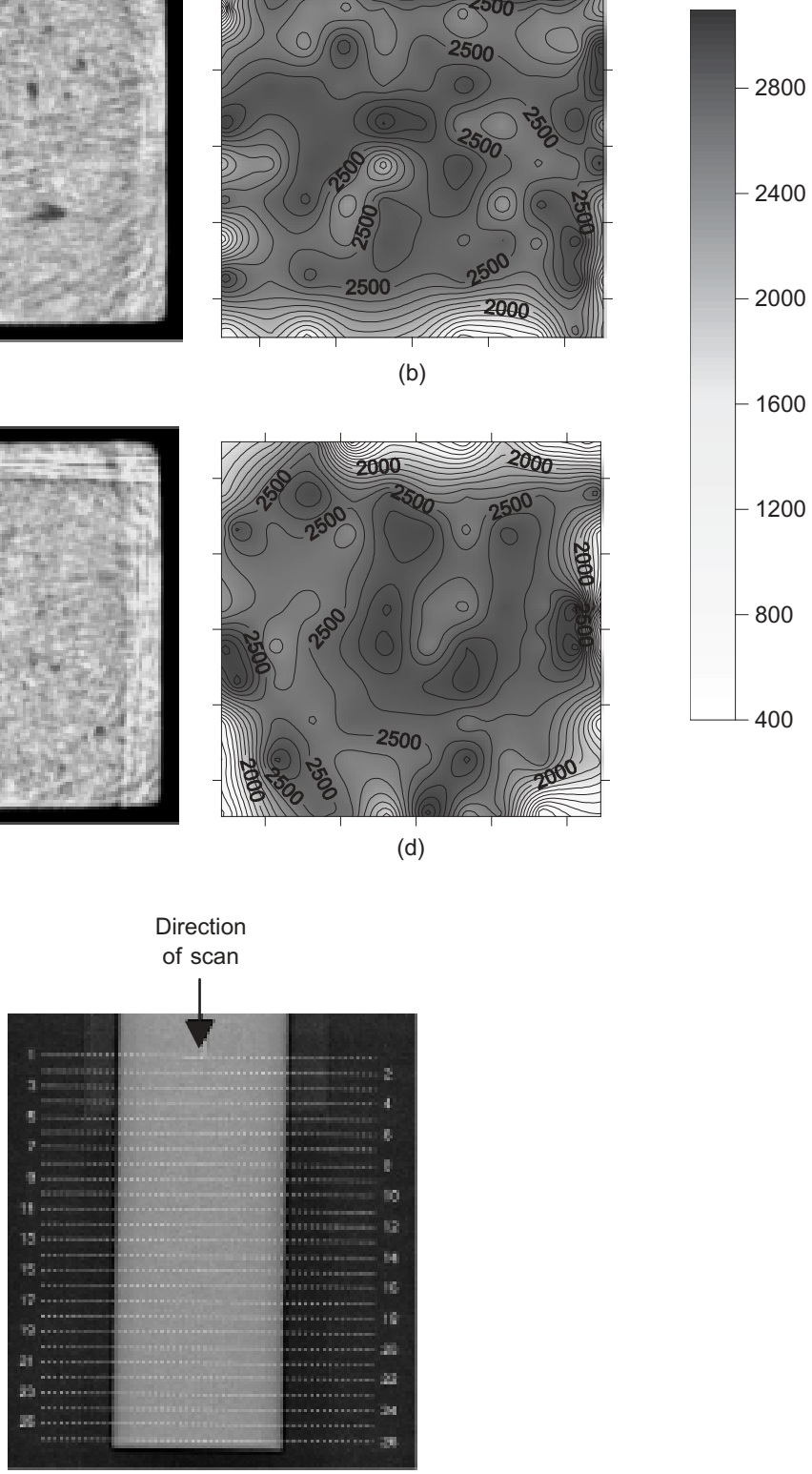

(e)

Fig. 1. CT captured images and contour plots showing the X-ray absorption densities for the beam cross-section $(100 \mathrm{~mm} \times 100 \mathrm{~mm}$ ). (a) CT image for slice 2, (b) contour plot for slice 2, (c) CT image for slice 18, (d) contour plot for slice 18 , and (e) plan view of the beam showing the location of each of the slices scanned (10 $\mathrm{mm}$ section spacing) and the direction of scanning

of fibres in each grid square calculated. Appropriate software (MATLAB 6.5) was used to plot the contours of fibre concentration in each section, for comparison with the corresponding contour plots obtained from the CT scanner.

Table 1 summarises the average numbers of fibres per $\mathrm{cm}^{2}$ that were counted under the optical microscope, for the several specimen configurations and sections under consideration. The number of fibres is calculated by multiplying the number of recorded features in the $0 \cdot 16-0 \cdot 32 \mathrm{~mm}$ range by one, the number of features recorded in the next higher range $(0 \cdot 32-$
$0.48 \mathrm{~mm}$ ) by two (two fibre diameters), and so on. From the number of fibres so calculated the average number of fibres per $\mathrm{cm}^{2}$ for each section was calculated.

From the known volume fractions of short ( $L=6 \mathrm{~mm})$ and long $(L=13 \mathrm{~mm})$ fibres in CARDIFRC $^{\mathbb{R}}$ mix I ( 4.5 and $1.5 \%$, respectively), it is easy to work out the number of short and long fibres in a certain volume of the material. The diameter of both types of fibre is equal to $d=0.16 \mathrm{~mm}$. Thus the number of $6 \mathrm{~mm}$ long fibres in $1000 \mathrm{~mm}^{3}$ is 373 and that of $13 \mathrm{~mm}$ long fibres in $1000 \mathrm{~mm}^{3}$ is 57 . 


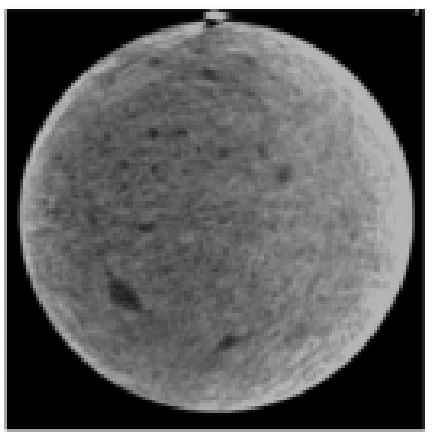

(a)

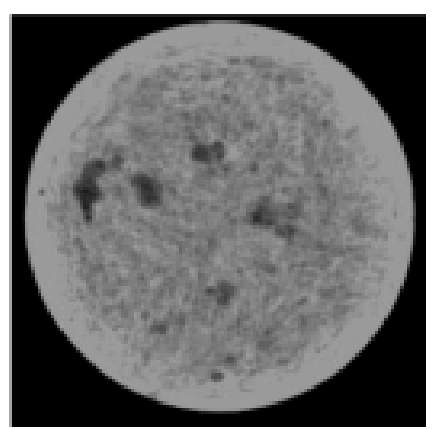

(c)

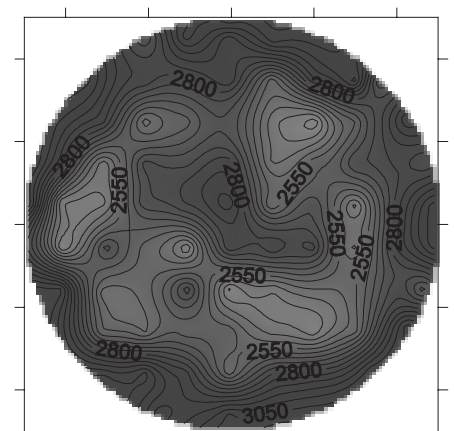

(b)

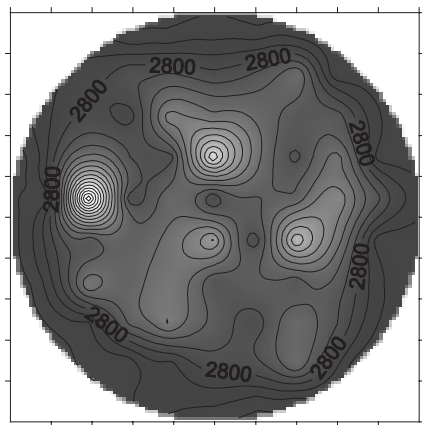

(d)

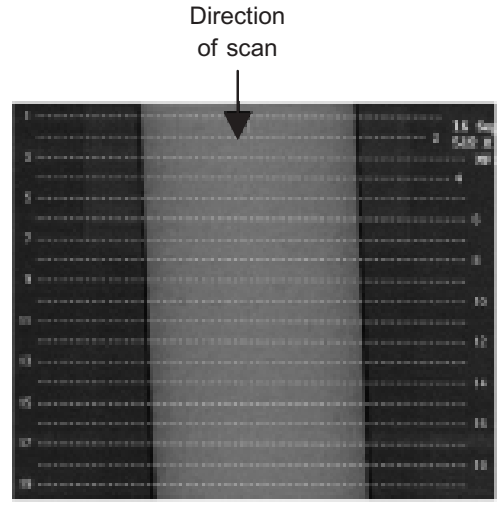

(e)

Fig. 2. CT captured images and contour plots showing the X-ray absorption densities for the cylinder (100 mm diameter). (a) CT image for slice 3, (b) contour plot for slice 3, (c) CT image for slice 10, (d) contour plot for slice 10, and (e) plan view of the cylinder showing the location of each of the slices scanned (10 mm section spacing) and the direction of scanning

The number of short and long fibres that will theoretically be seen in one cut section $(10 \mathrm{~mm} \times 10 \mathrm{~mm})$ of the particular volume of $1000 \mathrm{~mm}^{3}$ is given by the theory of geometric probability or more precisely by the solution of the so-called Buffon problem in three dimensions. ${ }^{6}$ The solution shows that when a needle (or line segment) of length $r$ is dropped at random in space containing a closed figure, then for a very large number of throws the following relation holds

$$
r \frac{E(h)}{E(c)}=4 \frac{\text { volume }}{\text { surface area }}
$$

Here, $E(h)$ is the number of hits; that is, the number of times each of the two end points of the line fall in the interior of the plane closed figure and $E(c)$ is the number of times the line intersects the perimeter of the figure. The result applies irrespective of whether the closed figure is convex or not. We shall use the above relation to calculate how many fibres of 6 and $13 \mathrm{~mm}$ length would cut a $10 \mathrm{~mm} \times 10 \mathrm{~mm}$ plane in a cube of side $10 \mathrm{~mm}$ (volume $1000 \mathrm{~mm}^{3}$ ). We note first that the numbers of 6 and $13 \mathrm{~mm}$ fibres in a $10 \mathrm{~mm}$ cube are 373 and 57, respectively, if the fibres are indeed randomly distributed, i.e. $E(h)+E(c)=373$ or 57 . From the above relation, it follows that the number of $6 \mathrm{~mm}$ fibres cut by a $10 \mathrm{~mm} \times 10 \mathrm{~mm}$ plane section is $E(c)=177$ and that of $13 \mathrm{~mm}$ fibres is $E(c)=38$. Thus, the theoretical maximum number of fibres

Magazine of Concrete Research, 2005, 57, No. 7 
(a)
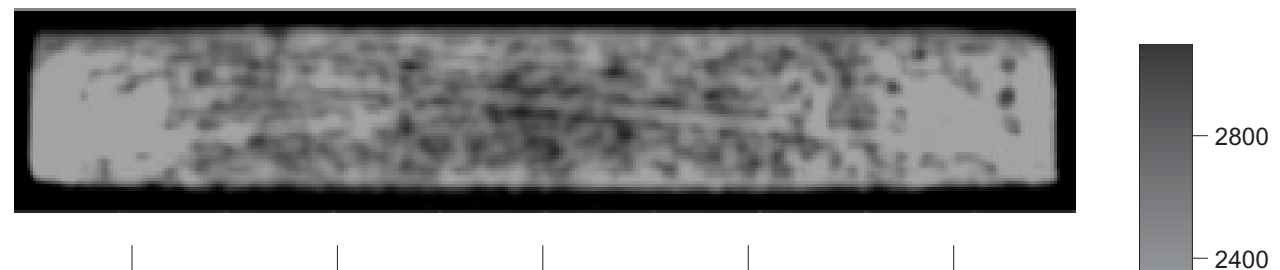

(b)

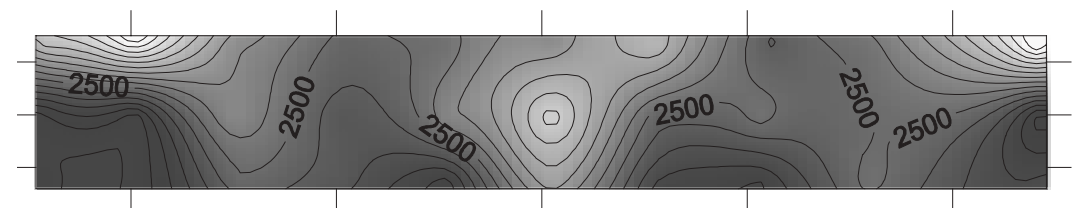

(c)

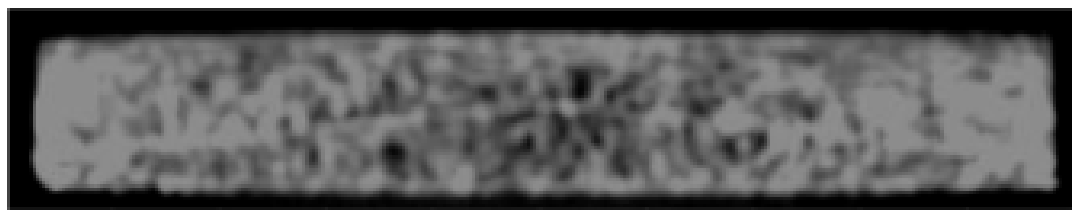

(d)

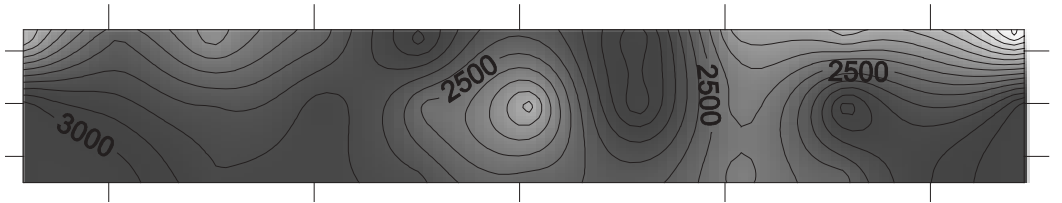

(e)

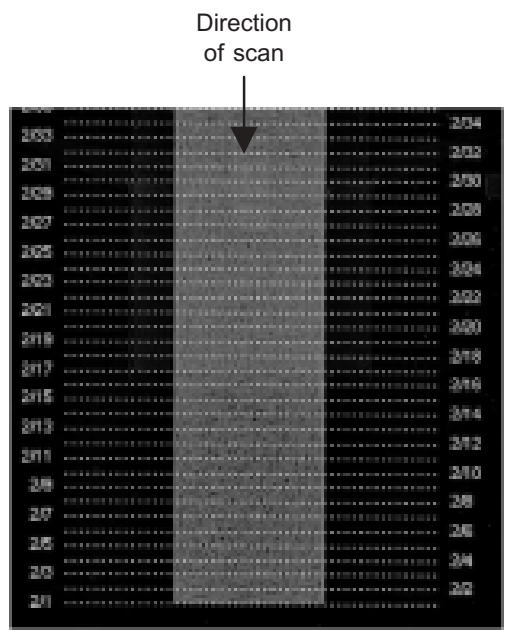

Fig. 3. CT captured images and contour plots showing the X-ray absorption densities for the $16 \mathrm{~mm}$ strip cross-section (100 mm width). (a) CT image for slice 2, (b) contour plot for slice 2, (c) CT image for slice 22, (d) contour plot for slice 22, and (e) plan view of the strip showing the location of each of the slices scanned (10 $\mathrm{mm}$ section spacing) and the direction of scanning

of both kinds that can appear in the cut section is $215 / \mathrm{cm}^{2}$.

The average number of fibres counted under the microscope $\left(150 / \mathrm{cm}^{2}\right)$ is smaller than this theoretical value. This lower number is, however, reasonable and expected for several reasons. First, as the specimens were cut by a $3 \mathrm{~mm}$ thick blade, the fibres embedded in the matrix to a depth of less than $3 \mathrm{~mm}$ would simply have fallen out during the cutting process. This would particularly be the case with $6 \mathrm{~mm}$ long fibres. Second, the image analyser would not account for fibres lying in the cut section or those that were in this plane prior to cutting. Third, there are many uncertainties in the image analysis count. These will be discussed below.
The contour plots of fibre concentration based on the image analysis fibre counts in $10 \mathrm{~mm} \times 10 \mathrm{~mm}$ grids were produced with MATLAB 6.5. These plots are shown in Figs $8-11$.

\section{Results and discussion}

The CT imaging contours show that for the beam specimen (Fig. 1) the fibres are reasonably uniformly distributed in each slice (section). However, the location of the section of scanning in relation to the ends and walls of the mould has a definite effect on the fibre distribution. Thus, the X-ray absorption density is lower 
(a)
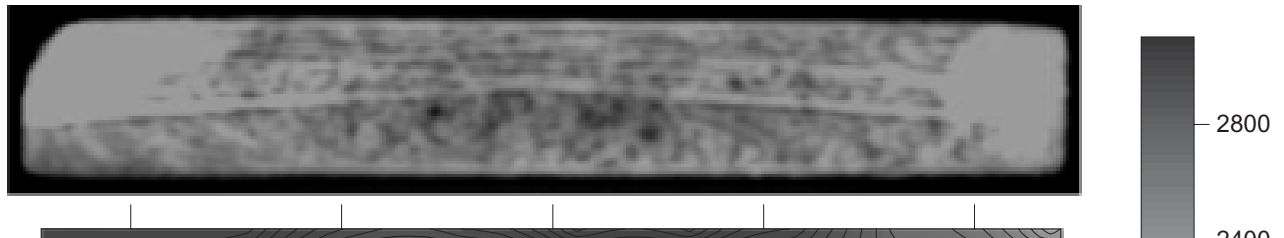

(b)

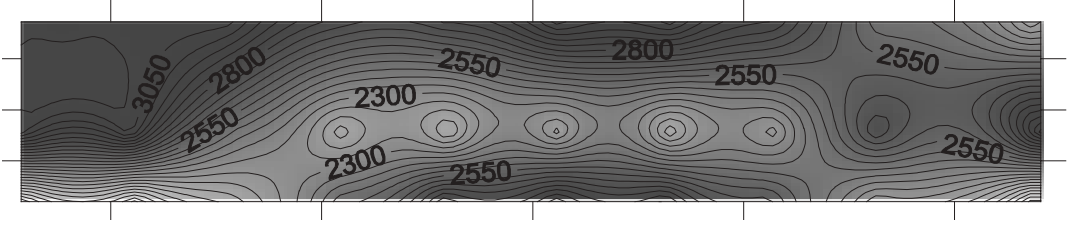

(c)

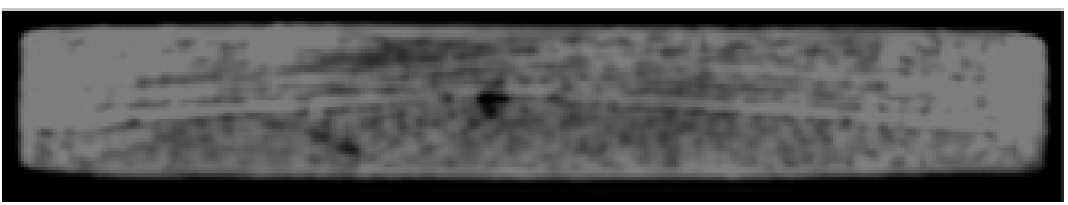

(d)
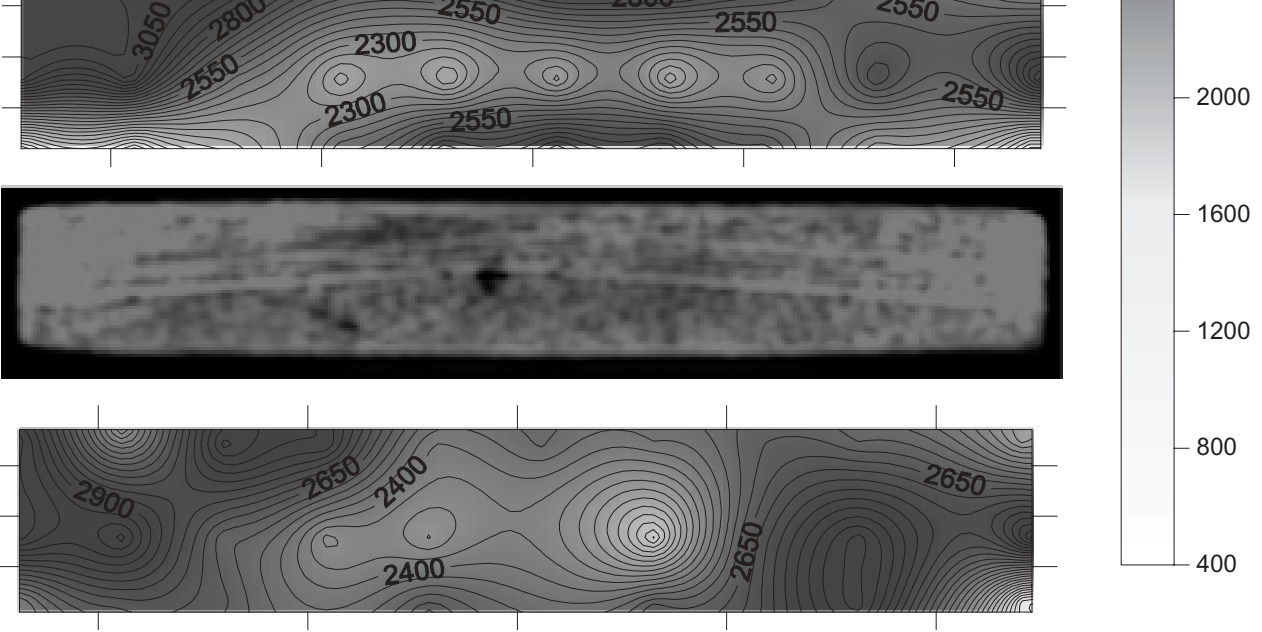

(e)

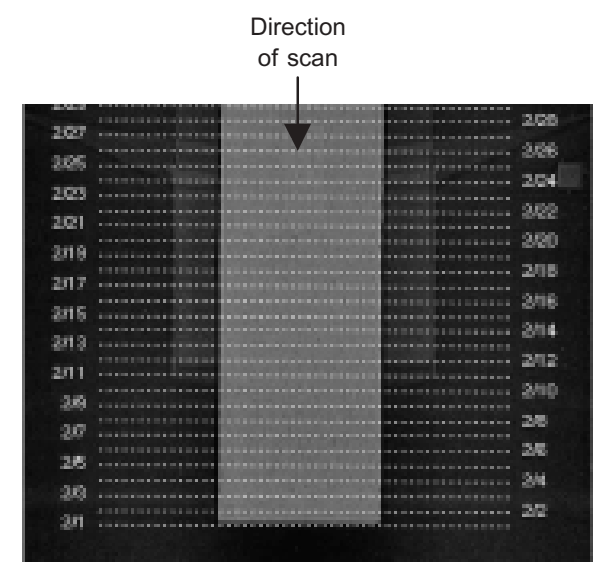

Fig. 4. CT captured images and contour plots showing the X-ray absorption densities for the $20 \mathrm{~mm}$ strip cross-section (100 mm width). (a) CT image for section 3, (b) contour plot for slice 3, (c) CT image for slice 20, (d) contour plot for slice 20, and (e) plan view of the strip showing the location of each of the slices scanned (10 mm section spacing) and the direction of scanning

(below 2000 Hounsfield units) in the bottom (casting face) and near the edges of slice 2 which is located close to the end of the mould.

There are also a few spots of lower X-ray absorption density (about 2500 Hounsfield units) in the interior. However, in slice 18 which is closer to the centre of the beam the effect from the mould walls is less noticeable, although the X-ray absorption density in the top of the slice close to the casting face is lower, as in slice 2 . The non-destructive CT images of the two beam slices are very similar to the fibre count contours obtained by sectioning and image analysis (Fig. 8). It should be noted that both slices were examined in the direction of casting.

X-ray absorption density contours (Fig. 2), as well as the fibre count contours (Fig. 9) of the two slices of the cylindrical specimen are very similar and show that there are patches of low fibre content in the interior of the slices. The images of the cut sections, prior to analysis (Fig. 5), show that these patches are areas of unhydrated material that has come loose during cutting. It is unsurprising that some unhydrated material should be present in the specimens given the very low waterto-binder ratio used. Moreover, as both slices were examined perpendicular to the direction of casting, the number of fibres along the circumference is likely to be more than that near the centre. This is due to the migration of some fibres from the centre to the circumference of the specimen under centrifugal action during vibratory compaction.

The thin strips of 16 and $20 \mathrm{~mm}$ thickness both show a lower concentration of fibres near the cast faces (Figs 10 and 11) and ends of the mould, as in the beam specimen above.

Magazine of Concrete Research, 2005, 57, No. 7 


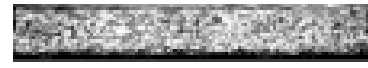

$16 \mathrm{~mm}$ strip slice 22

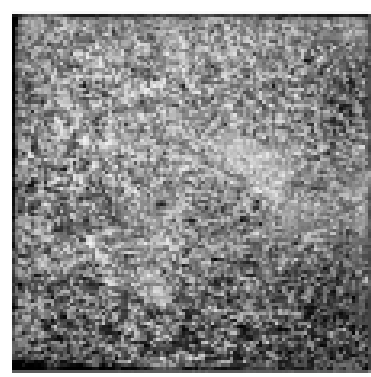

Beam slice 2

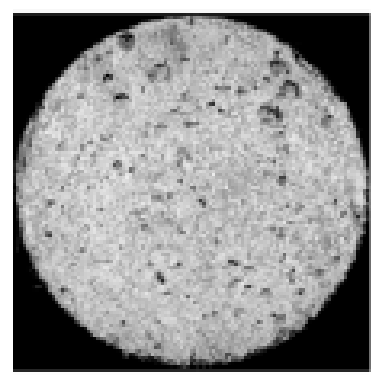

Cylinder slice 3

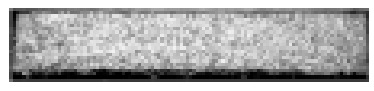

$20 \mathrm{~mm}$ strip slice 20

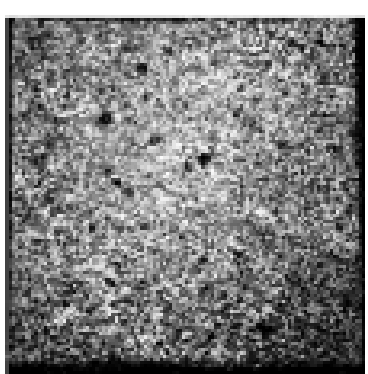

Beam slice 18

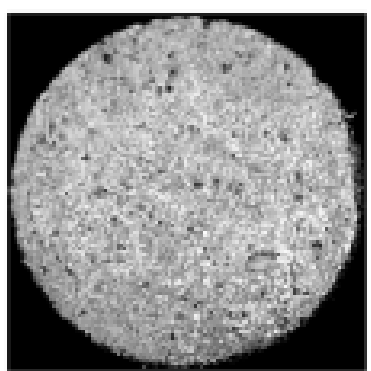

Cylinder slice 10
Fig. 5. CARDIFRC $C^{\circledR}$ specimen sections used for image analysis
Table 1. Average number of fibres resulting from image analysis of specimen sections

\begin{tabular}{l|c}
\hline & $\begin{array}{c}\text { Average number of fibres per } \\
\mathrm{cm}^{2}\end{array}$ \\
\hline $16 \mathrm{~mm}$ strip: slice 2 & 132 \\
$16 \mathrm{~mm}$ strip: slice 22 & 157 \\
$20 \mathrm{~mm}$ strip: slice 3 & 128 \\
$20 \mathrm{~mm}$ strip: slice 20 & 178 \\
$100 \mathrm{~mm} \times 100 \mathrm{~mm}$ beam: slice 2 & 173 \\
$100 \mathrm{~mm} \times 100 \mathrm{~mm}$ beam: slice 18 & 172 \\
$100 \mathrm{~mm}$ cylinder: slice 3 & 125 \\
$100 \mathrm{~mm}$ cylinder: slice 10 & 134 \\
Average & 150 \\
\hline
\end{tabular}

In all specimen types examined, the differences in the fibre distribution revealed by CT imaging and image analysis are a result of several factors connected with the casting and compaction procedures used to make the specimens and with the peculiarities of the detection methods themselves. The most important factors are the following.

(a) The fibre orientation can be altered by the walls of the mould during casting and compaction. Further

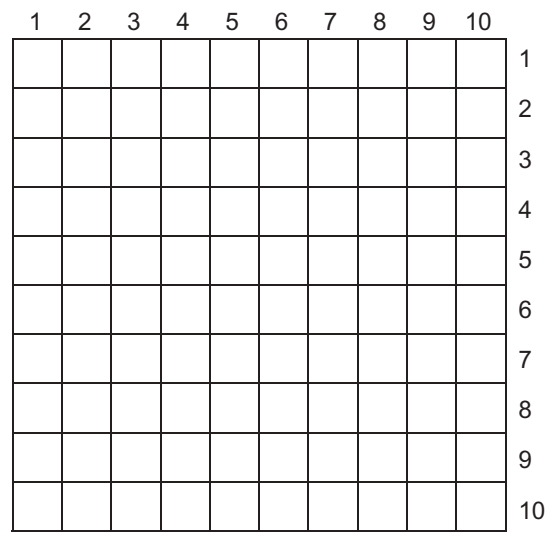

(a)

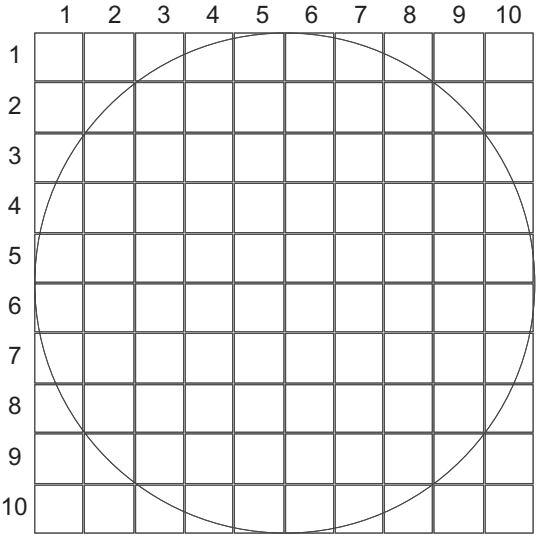

(b)

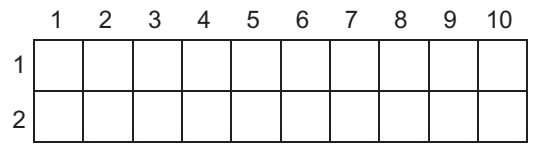

(c)

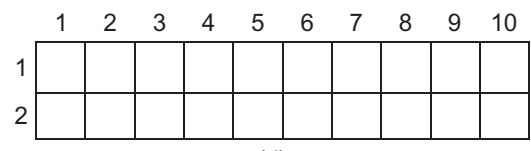

(d)

Fig. 6. $10 \mathrm{~mm} \times 10 \mathrm{~mm}$ grids used for image analysis. (a) $100 \mathrm{~mm} \times 100 \mathrm{~mm}$ beam, (b) $100 \mathrm{~mm}$ diameter cylinder, (c) $20 \mathrm{~mm}$ thin strip and (d) $16 \mathrm{~mm}$ thin strip (the grid in row two is only $6 \mathrm{~mm} \times 10 \mathrm{~mm}$ ) 


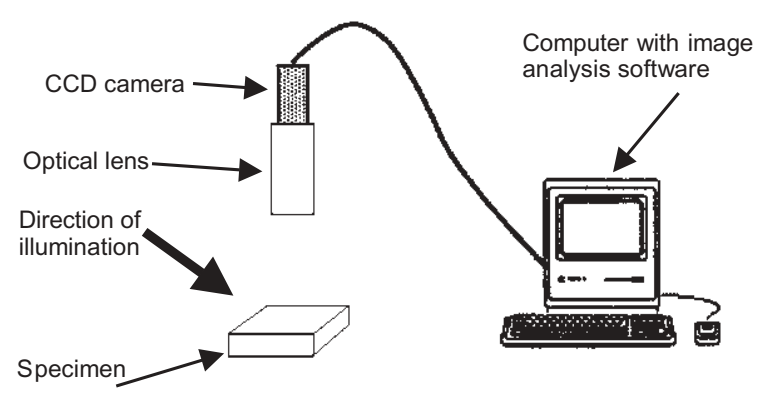

Fig. 7. Image acquisition system

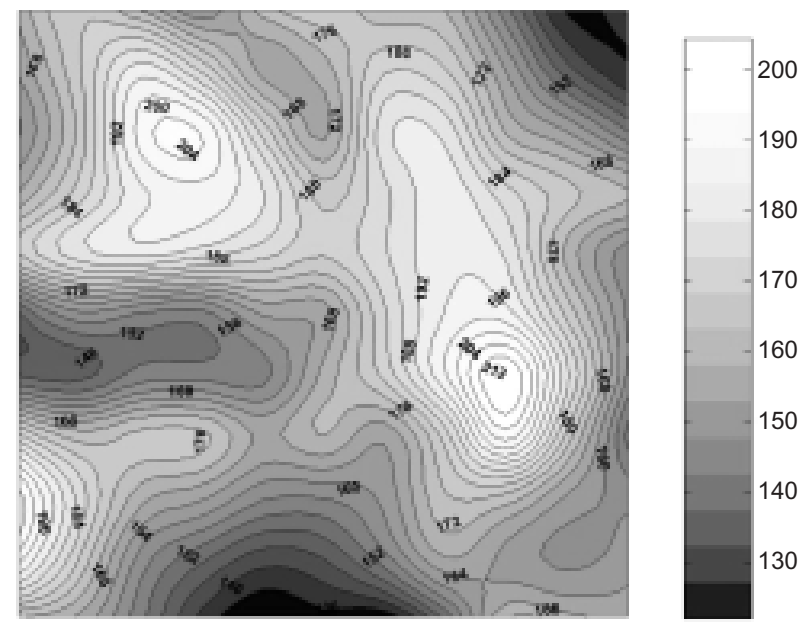

(a)

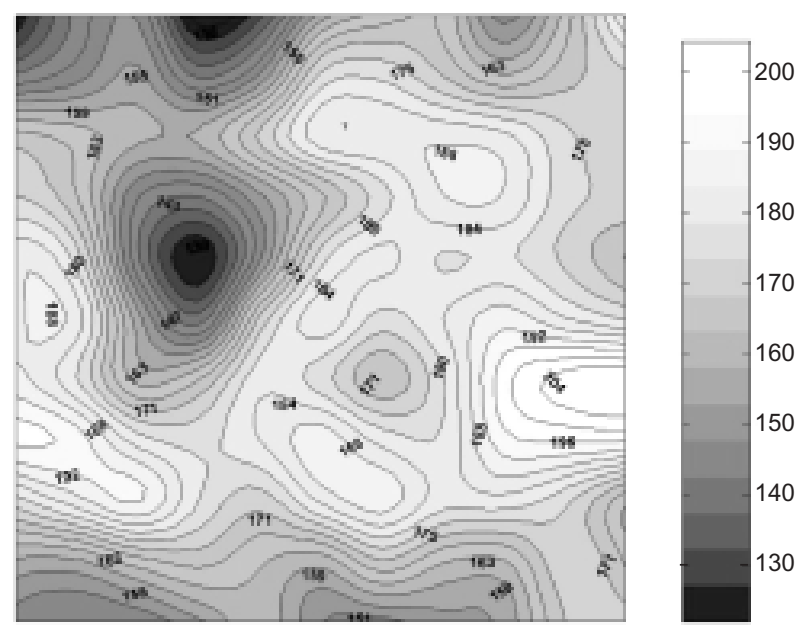

(b)

Fig. 8. Contour plots showing the fibre distribution for the $100 \mathrm{~mm} \times 100 \mathrm{~mm}$ beam cross-section for (a) slice 2 and (b) slice 18

alteration can be induced by the float used to finish the casting face of the specimen.

(b) The fibres near the edges of the specimen are more likely to be bent during cutting. These fibres will
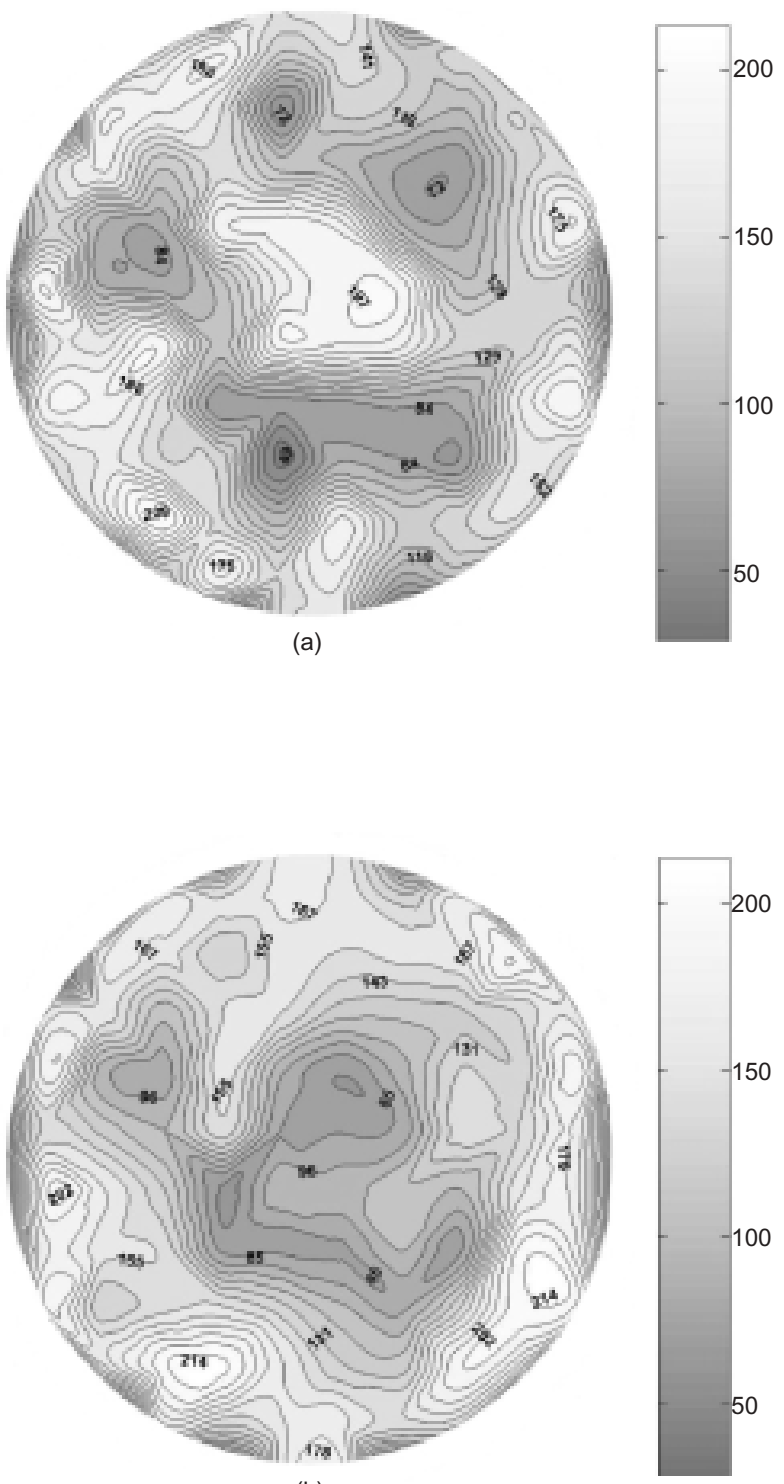

(b)

Fig. 9. Contour plots showing the fibre distribution for the cylinder (100 mm diameter) cross-section for (a) slice 3 and (b) slice 10

not be illuminated by the light source during image analysis and will thus not enter the fibre count. This effect will be more prominent in thin specimens.

(c) As the diamond saw used for cutting the specimens has to be water cooled, it is not possible to collect the debris to estimate how many fibres have been dislodged during cutting.

(d) The sections examined by CT imaging were removed from those examined by image analysis by the thickness of the saw blade $(3 \mathrm{~mm})$. Thus some differences in the fibre distributions are to be expected.

(e) The CT scans are likely to enhance the X-ray absorption density, namely the fibre concentration, near the edges of the sections. This is believed to be due to the so-called beam hardening which

Magazine of Concrete Research, 2005, 57, No. 7 
(a)

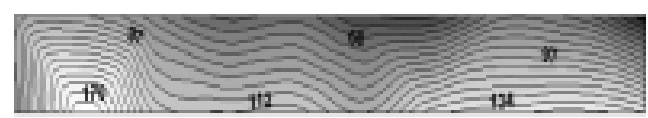

(b)

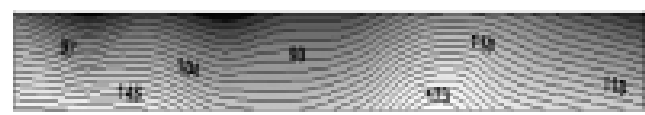

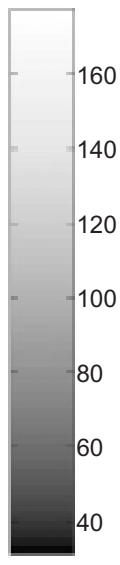

Fig. 10. Contour plots showing the fibre distribution for the $16 \mathrm{~mm}$ strip cross-section (100 $\mathrm{mm}$ width) for (a) slice 2 and (b) slice 22

(a)

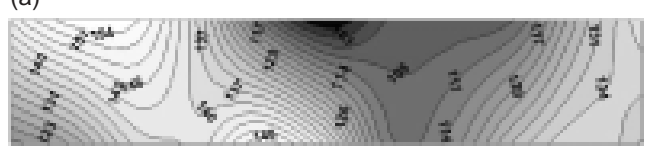

(b)

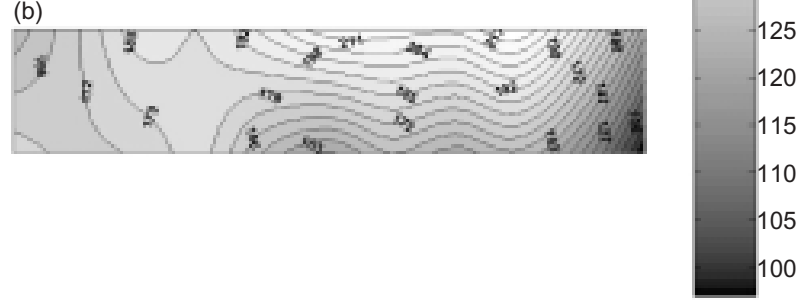

Fig. 11. Contour plots showing the fibre distribution for the $20 \mathrm{~mm}$ strip cross-section (100 $\mathrm{mm}$ width) for (a) slice 3 and (b) slice 20

creates 'arcs of white light' at the edges of the sections (Figs 1-4). This is a common problem in medical imaging (S. Lawrence, personal comm., 2002) caused by a particularly dense or thick material casting a shadow over the material behind it. It is most apparent when the section is thin and long (Figs 3 and 4).

$(f)$ X-ray absorption density will be large in regions where the fibres are clustered (Fig. 12) and the nearest-neighbour distance is greatly reduced. The fibre density in these regions will be higher than in the surrounding area.

( $g$ ) Depending on the shape of the fibre cluster, the feature detected by the image analyser may be incorrectly interpreted by the software leading to an erroneous fibre count in a particular grid. This can be partially avoided by a thorough visual inspection of the analysed section.

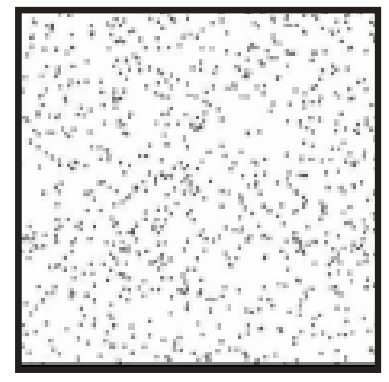

(a)

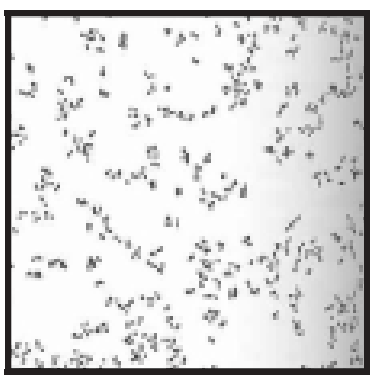

(b)
Fig. 12. Uniform distribution of fibres (a) and clustered distribution of fibres (b)

(h) Fibres may be double-counted or missed altogether if there is a mistake in shifting the section under the optical microscope from one grid to the next.

(i) Cutting the specimens is likely to loosen and dislodge unhydrated cement-silica powder from the section together with any loose fibres. This will particularly affect the fibre count in the interior of thicker specimens (see beam and cylinder specimen sections in Fig. 5).

Despite the many potential sources of error listed above, a careful examination of the X-ray absorption density contours (Figs 1-4) and the corresponding fibre count contours (Figs 8-11) reveals a reasonable correlation between the two measurements in the larger sections examined by the two techniques. In the thin strips the correlation is poor for the reasons explained above. For a better comparison of the X-ray absorption density with the actual fibre count, contour plots of two beam and two cylinder sections have been plotted side by side in Figs 13 and 14. Moreover, the actual $\mathrm{X}$-ray absorption densities at grid centres have been plotted against the fibre counts per grid for these sections in Figs 15 and 16. It is clear from these figures that the X-ray absorption density correlates reasonably linearly with the fibre count in the thicker specimens, irrespective of their shapes.

\section{Conclusions}

(a) The contour plots of the X-ray absorption density faithfully reproduce the CT scans. Attention however needs to be paid to the beam hardening effect.

(b) The contour plots from both techniques are a useful visual aid for assessing the distribution of fibres achieved by the mixing and compaction procedures used for making CARDIFRC ${ }^{\circledR}$ products. Although both techniques reveal that the fibre distribution is reasonably uniform in larger sections, the need to achieve a better distribution in thinner sections is also highlighted. 


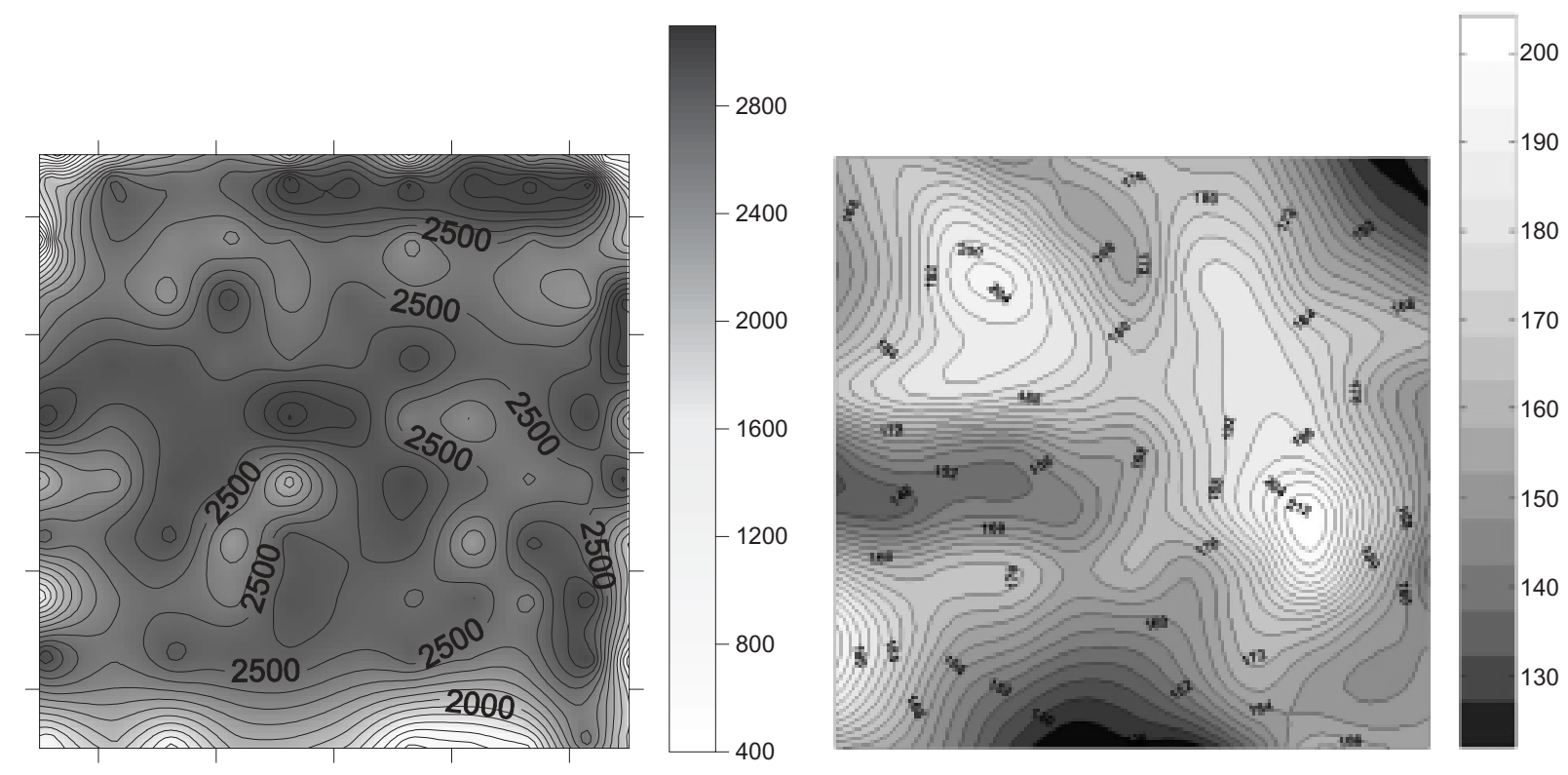

(a)

(b)

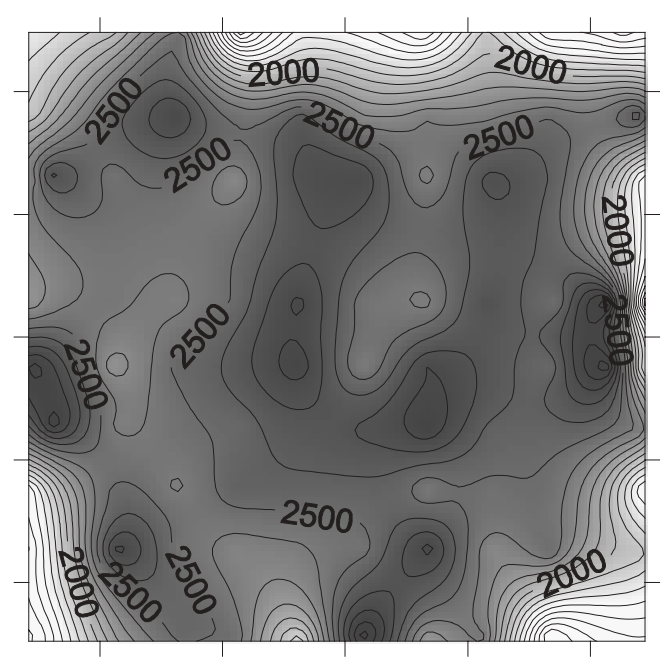

(c)

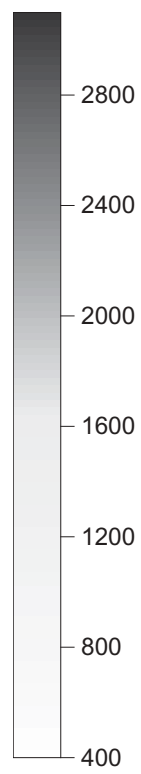

400

Fig. 13. Contour plots showing the X-ray absorption densities (a) and (c), and the fibre count (b) and (d) for the beam slices 2 and 18 , respectively 


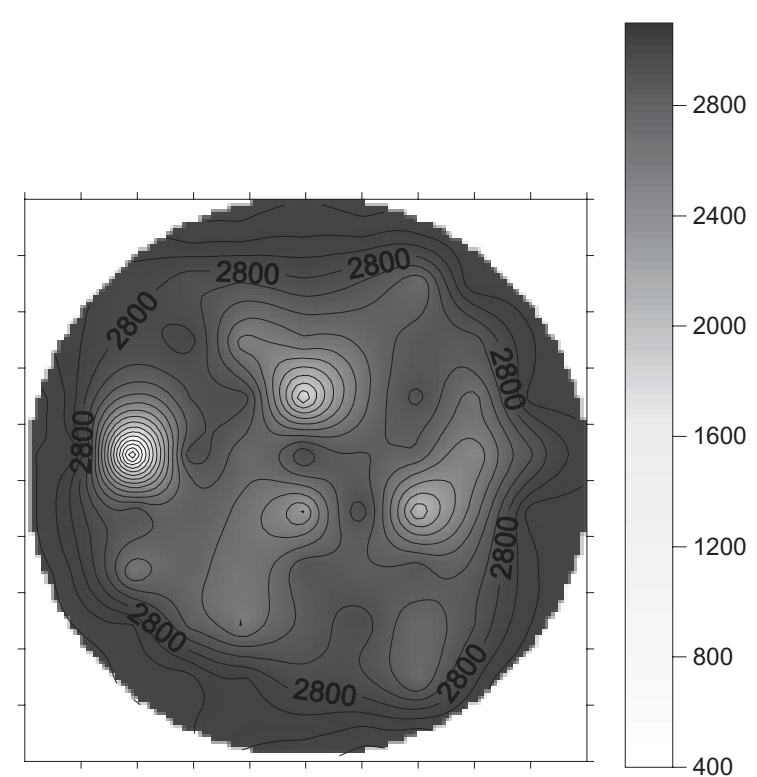

(a)

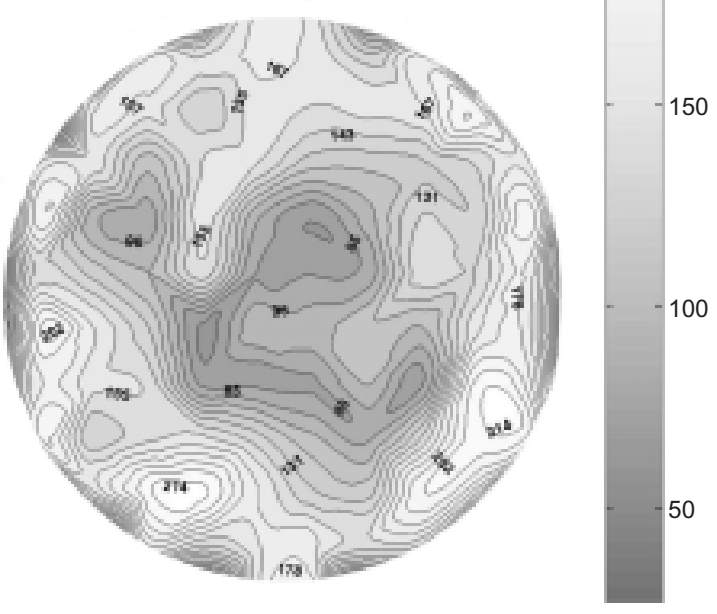

(b)

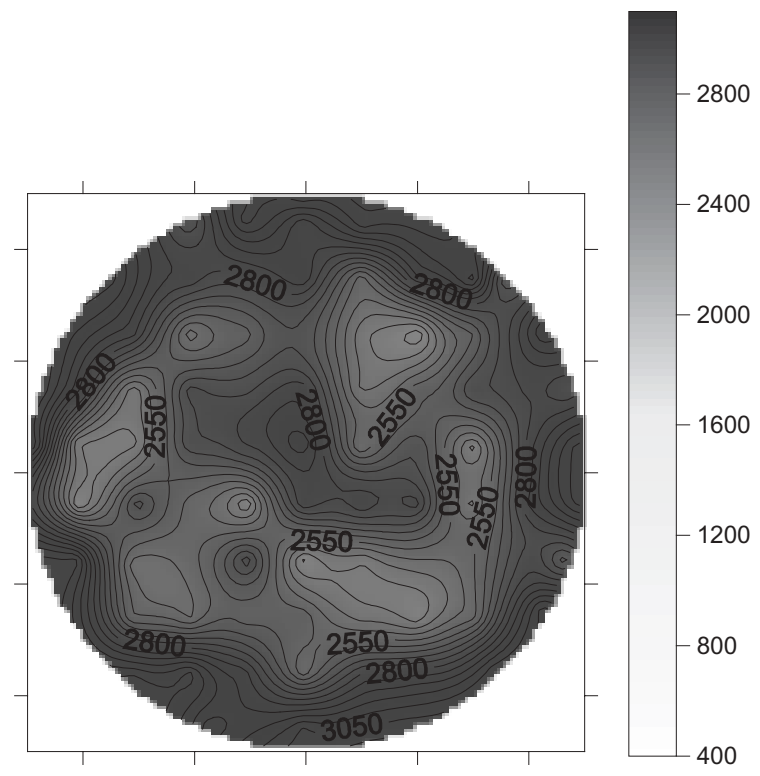

(c)

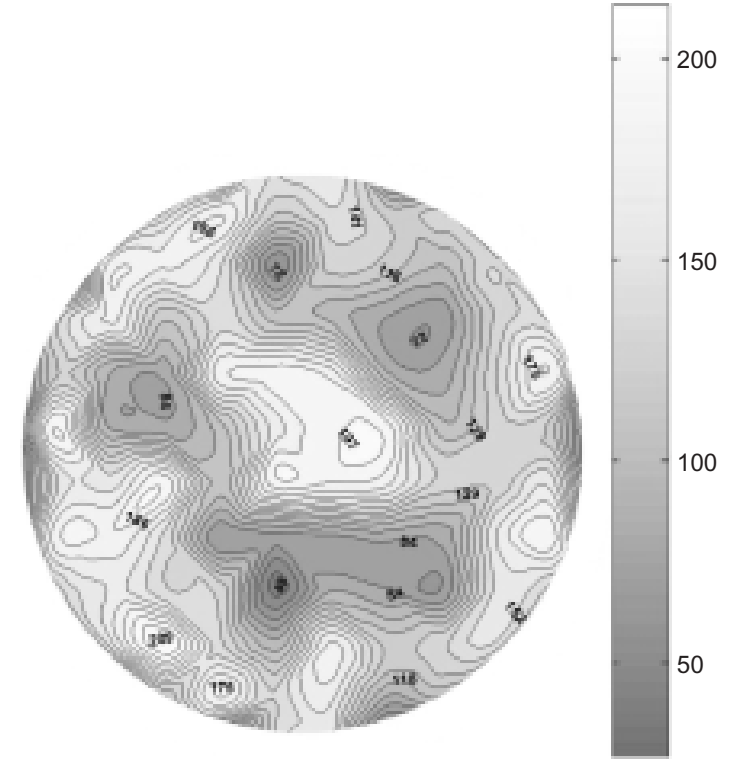

(d)

Fig. 14. Contour plots showing the X-ray absorption densities (a) and (c), and the fibre count (b) and (d) for the cylinder slices 10 and 3 , respectively 


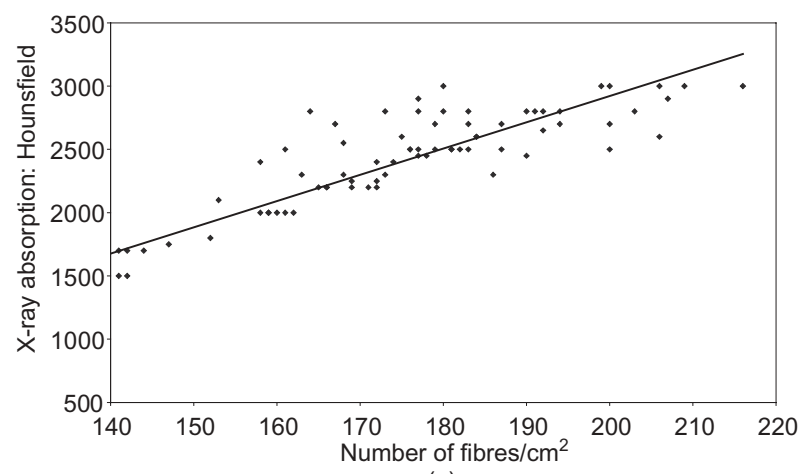

(a)

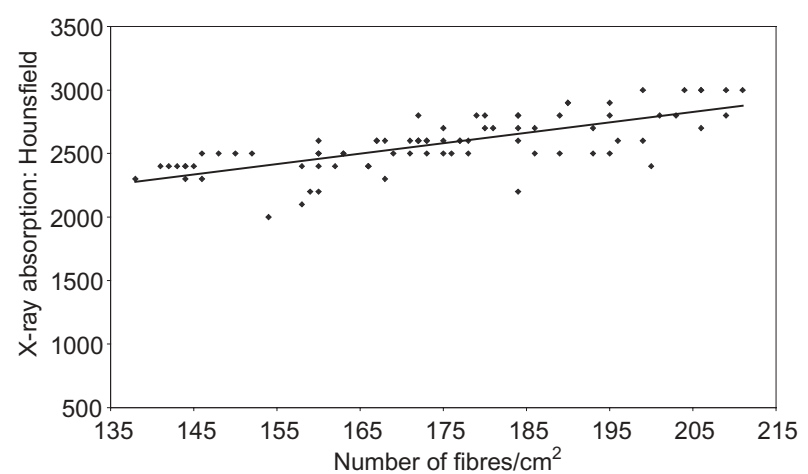

(b)

Fig. 15. X-ray absorption density versus number of fibres per $\mathrm{cm}^{2}$ in (a) beam slice 2 and (b) beam slice 18

(c) In thicker sections the X-ray absorption density plots are reasonably linearly related to the actual fibre concentration. Thus the non-destructive CT imaging technique can be used to control the quality of products in a factory without the need for destructive testing.

The final companion part (Part III) of this three-part paper will compare the measured mechanical properties of CARDIFRC ${ }^{\mathrm{B}}$ with theoretical predictions.

\section{Acknowledgements}

We thank the staff at Velindre Hospital, Cardiff, for their assistance with the CT scanning. This work is supported by the UK EPSRC grant GR/R11339.

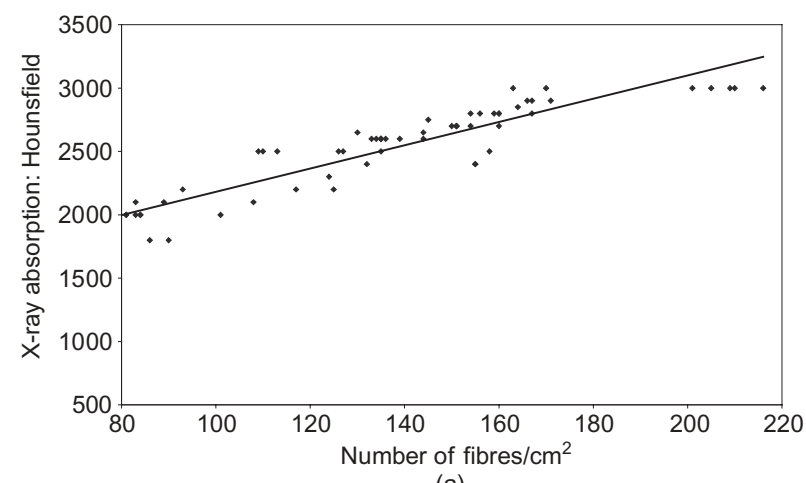

(a)

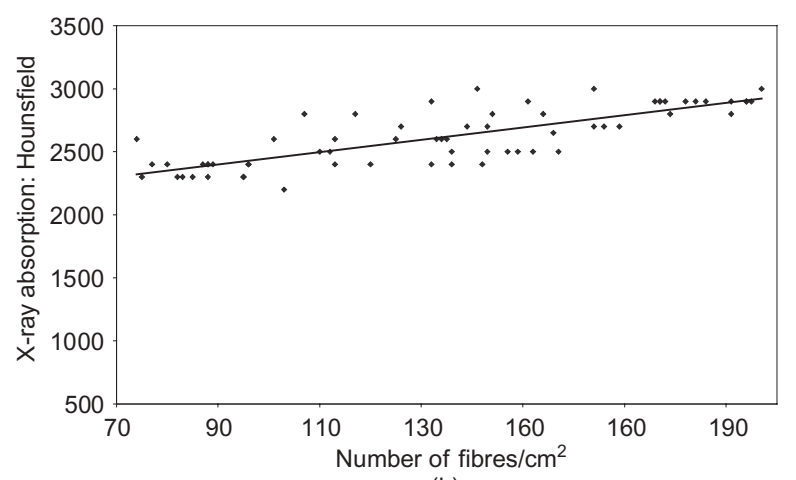

(b)

Fig. 16. X-ray absorption density vs. number of fibres per $\mathrm{cm}^{2}$ in (a) cylinder slice 10 and (b) cylinder slice 3

\section{References}

1. Karihaloo B. L. and JefFerson A. D. Looking into concrete. Magazine of Concrete Research, 2001, 53, No. 2, 135-147.

2. Karihaloo B. L., Benson S. D. P. and Alaee F. J. CarDI$F R C^{\mathbb{R}}$ Patent Number GB 0109686.6, 2001.

3. Benson S. D. P. CARDIFRC ${ }^{\mathrm{B}}$ - Development and Constitutive Behaviour. PhD Thesis, University of Wales, Cardiff, 2003.

4. Sullivan A. P. The Effect of Hydro-thermal Curing at $90^{\circ} \mathrm{C}$ and the Use of $12 \mathrm{~mm}$ Steel Fibres on Reactive Powder Concrete. MSc Thesis, University of Wales, Cardiff, 1999.

5. Alaee F. J., Benson S. D. P and Karihaloo B. L. Highperformance fibre-reinforced cementitious composites for retrofitting. In Proceedings of the ACUN-3 International Conference Technology Convergence in Composite Applications (BANDYOPadHyay S., Gowripalan N. and Drayton N. (eds.)), University of New South Wales, 2001, pp. 8-17

6. Solomon H. Geometric Probability, Society for Industrial and Applied Mathematics, USA, 1978.

Discussion contributions on this paper should reach the editor by 1 March 2006 